\title{
SEMI-ALGEBRAIC GROUPS AND THE LOCAL CLOSURE OF AN ORBIT IN A HOMOGENEOUS SPACE \\ BY \\ MORIKUNI GOTO
}

\begin{abstract}
Let $L$ be a topological group acting on a locally compact Hausdorff space $M$ as a transformation group. Let $m$ be in $M$. A subset $Q$ of $M$ is called the local closure of the orbit $L m$ if $Q$ is the smallest locally compact invariant subset of $M$ with $m \in Q$. A partition
\end{abstract}

$$
M=\bigcup_{\lambda \in \Lambda} Q_{\lambda}, \quad Q_{\lambda} \cap Q_{\mu}=\varnothing \quad(\lambda \neq \mu)
$$

is called an LC-partition of $M$ with respect to the $L$ action if each $Q_{\lambda}$ is the local closure of $\mathbf{L m}$ for any $m$ in $Q_{\lambda}$.

TheOREM. Let $G$ be a connected Lie group, and let $A$ and $B$ be subgroups of $G$ with only finitely many connected components. Suppose that $B$ is closed. Then the factor space $G / B$ has an LC-partition with respect to the $A$ action.

1. Introduction. Let $G$ be a locally compact topological group, and let $A$ and $B$ be subgroups of $G$. A subset $P$ of $G$ is said to be $(A, B)$-invariant if $A P B=\{a p b ; a \in A, p \in P, b \in B\}=P$. In this case the direct product topological group $A \times B$ acts on the underlying space of $G$ as a transformation group by

$$
(a, b) g=a g b^{-1} \quad \text { for } g \in G, \quad(a, b) \in A \times B,
$$

and $P$ is $(A, B)$-invariant if and only if $P$ is invariant under $A \times B$. For $g$ in $G$, the double coset $A g B$ is the orbit of the transformation group, passing through $g$. In this setting, we shall give some definitions.

Definition 1. Let $g$ be in $G$, and suppose that there exists a minimal, locally compact, $(A, B)$-invariant subset $P$ containing $g$. Then the set $P$ is said to be the local closure of the double coset $A g B$.

Since the intersection of two locally compact sets is locally compact, the local closure of $A g B$ is unique, if it exists.

Definition 2. Suppose that $G$ has a partition

$$
G=\bigcup_{\lambda \in \Lambda} P_{\lambda}, \quad P_{\lambda} \cap P_{\mu}=\varnothing \quad(\lambda \neq \mu),
$$

where $\Lambda$ is a set of indices, such that for each $\lambda \in \Lambda$, the set $P_{\lambda} \neq \varnothing$ is the

Received by the editors May 15, 1977 and, in revised form, November 22, 1977.

AMS (MOS) subject classifications (1970). Primary 57E20; Secondary 20G20, 22D05. 
local closure of $A g B$ for every $g$ in $P_{\lambda}$. Then the partition (clearly unique) is called the LC-partition of $G$ with respect to the pair $(A, B)$.

Throughout the paper, the identity element of a group in question is always denoted by $e$; for a topological group $L$, the identity component, i.e. the connected component containing $e$, will be denoted by $L_{e}$.

A topological group $L$ is said to be compactly, finitely, or countably connected if the factor group $L / L_{e}$ is compact, finite, or countable, respectively.

The following theorem will be established in this paper:

TheOREM I. Let $G$ be a connected Lie group, and let $A$ and $B$ be finitely connected subgroups of $G$. Then $G$ has an $L C$-partition with respect to $(A, B)$.

Next, we shall consider transformation groups.

Definition 3. Let $L$ be a topological group acting on a locally compact Hausdorff space $M$ as a transformation group. Let $m$ be in $M$. A subset $Q$ of $M$ is called the local closure of the orbit $L m$ if $Q$ is the smallest locally compact, invariant subset of $M$ with $m \in Q$. Also, a partition

$$
M=\bigcup_{\lambda \in \Lambda} Q_{\lambda}, \quad Q_{\lambda} \cap Q_{\mu}=\varnothing \quad(\lambda \neq \mu),
$$

is called the LC-partition, of $M$ with respect to the $L$ action, if each $Q_{\lambda}$ is the local closure of $L m$ for any $m$ in $Q_{\lambda}$.

Let $G$ be a locally compact group, and $B$ a closed subgroup of $G$. Let $M=G / B=\{g B ; g \in G\}$ denote the factor space, and $\pi: G \ni g \mapsto g B \in$ $M$ the natural map. Let $A$ be a subgroup of $G$. Then $A$ acts on the homogeneous space $M$ by $a \pi(g)=\pi(a g)$ for $a \in A$ and $g \in G$. Now, it is easy to see that for any $g \in G$, a subset $Q$ of $M$ is the local closure of the orbit $A \pi(g)=\pi(A g)$ if and only if $\pi^{-1} Q$ is the local closure of the double coset $A g B$. Therefore from Theorem I we have

TheOREM I'. Let $G$ be a connected Lie group, and let $A$ and $B$ be finitely connected subgroups. Suppose that $B$ is closed, and let $M=G / B$ be the factor space. Then $M$ has an $L C$-partition with respect to the $A$ action.

Throughout the paper, for a subset $X$ of a topological space, we let $\bar{X}$ denote the closure of $X$.

Let $L$ be a topological group, and let $A$ and $B$ be subgroups of $L$. If a locally compact subset $P$ of $L$ is $(A, B)$-invariant, then $P$ is $(\bar{A}, \bar{B})$-invariant, see (2.1) below. Also if $B$ is finitely connected, then so is $\bar{B}$; see (2.3). Therefore Theorem I' implies Theorem I, i.e. Theorem I and Theorem I' are equivalent to each other.

Next, in Theorem $I^{\prime}$ we let $A$ be an abelian group and $B$ a connected 
group. In this case, each local closure has an extremely simple topological structure.

Theorem II. Let $G$ be a connected Lie group. Let $A$ be a finitely connected, abelian subgroup and $B$ a closed connected subgroup of $G$. Then the local closure of any orbit of the transformation group $A$ acting on $M=G / B$ is homeomorphic with the underlying space of a certain abelian Lie group.

If, in particular, $A$ is a one-parameter subgroup, then for any $m \in M$, the local closure of the orbit Am is homeomorphic with either the real line $\mathbf{R}$ or a toral group.

In order to explain the outline of our proof, we shall introduce a notation of an LC-family of subgroups. When a topological space $X$ is a union of countably many compact subsets, $X$ is said to be $\sigma$-compact.

Definition 4. Let $G$ be a locally compact, $\sigma$-compact topological group. A set $\mathscr{F}$ of subgroups of $G$ is said to form an LC-family of $G$ if the following conditions are satisfied:

(1) Any member of $\mathscr{F}$ is closed.

(2) $G \in \mathscr{F}$, and for any nonempty subset $\mathscr{F}^{\prime}$ of $\mathscr{F}$, the intersection of all members of $\mathscr{F}^{\prime}$ is contained in $\mathscr{F}$.

By (2), to any subgroup $H$ of $G$, we can associate the $\mathscr{F}$-hull $\mathscr{F}(H)$, which is the smallest member of $\mathscr{F}$ containing $H$. By $(1), \mathscr{F}(\bar{H})=\mathscr{F}(H)$.

(3) If $F \in \mathscr{F}$ and $g \in G$, then $g F g^{-1} \in \mathscr{F}$.

(4) For $F_{1}$ and $F_{2}$ in $\mathscr{F}$, the double coset $F_{1} F_{2}$ is a locally compact set.

(5) Suppose $F_{1}$ and $F_{2}$ are closed subgroups of $G$ such that $F_{1} \supset F_{2}$ and the factor space $F_{1} / F_{2}$ is compact and totally disconnected. If one of $F_{1}$ and $F_{2}$ is in $\mathcal{F}$, then so is the other one.

(6) If $H$ is a closed connected subgroup of $G$, then $\mathscr{F}(H)$ is connected, and the commutator subgroup of $H$ coincides with the commutator subgroup of $\mathscr{F}(H)$.

(7) If $H$ is a compactly connected, abelian subgroup of $G$, then $\mathscr{F}(H)$ is abelian.

Suppose that $G$ has an LC-family $\mathscr{F}$. Let $A$ and $B$ be subgroups of $G$, and put $A^{*}=\mathscr{F}(A)$ and $B^{*}=\mathscr{F}(B)$. By (3) and (4), every double coset $A^{*} g B^{*}$ is locally compact, and $G=\cup A^{*} g B^{*}$ gives a partition of $G$ into locally compact, $(A, B)$-invariant subsets. Hence we only have to study the action of $A \times B$ in each $A^{*} g B^{*}$. On the other hand, since $A^{*} \times B^{*}$ is a locally compact, $\sigma$-compact group, the map

$$
\xi_{g}: A^{*} \times B^{*} \ni(\alpha, \beta) \mapsto \alpha g \beta^{-1} \in A^{*} g B^{*}
$$

is (continuous and) open, see (2.4), and induces a homeomorphism between the factor space $A^{*} \times B^{*} / D(g)$ and $A^{*} g B^{*}$, where $D(g)$ is the isotropy 
subgroup of $A^{*} \times B^{*}$ at $g$. We see easily that a subset $P$ of $A^{*} g B^{*}$ is locally compact if and only if $\xi_{g}^{-1} P$ is so, and $P$ is $(A, B)$-invariant if and only if $\xi_{\mathrm{g}}{ }^{-1} P$ is $(A \times B, D(g))$-invariant. Thus the existence of an LC-partition of $G$ with respect to $(A, B)$, is reduced to the existence of an LC-partition of $A^{*} \times B^{*}$ with respect to $(A \times B, D(g))$ for every $g \in G$.

Next, let us consider the double coset decomposition

$$
A^{*} \times B^{*}=\cup(A \times B) x D(g) .
$$

When $A$ and $B$ are closed connected subgroups, $A \times B$ is a normal subgroup of $A^{*} \times B^{*}$, and $(A \times B) D(g)$ is a group. Therefore each double coset is a coset. It is easy to see that

$$
A^{*} \times B^{*}=\cup x \overline{(A \times B) D(g)}
$$

gives the LC-partition. When $A$ and $B$ are compactly connected or when $A$ is abelian, etc., we can modify the connected case and can study the $(A \times B) \times$ $D(g)$ action on $A^{*} \times B^{*}$ in details using (5), (6) and (7). The results following from the existence of an LC-family are given in Theorem III, \$2.

Now the problem boils down to the following:

Which groups have an LC-family?

First we consider $\mathrm{GL}(n, \mathbf{R})$. Let $\mathbb{Q}^{*}$ denote the set of all algebraic groups in $\mathrm{GL}(n, \mathbf{R}) . \mathbb{Q}^{*}$ is almost an LC-family, but does not satisfy (5), (6). We shall call a subgroup $A$ of $\mathrm{GL}(n, \mathbf{R})$ pre-algebraic if $A$ is an open subgroup of a suitable algebraic group. Let $\mathbb{Q}$ denote the set of all pre-algebraic groups in $\mathrm{GL}(n, \mathbf{R})$. Then $\mathbb{Q}$ is known to be an LC-family. For a subgroup $H$ of $\mathrm{GL}(n, \mathbf{R})$, let $\mathbb{Q}(H)$ denote the pre-algebraic hull (= $Q$-hull) of $H$.

A notion of (connected) semi-algebraic groups was introduced by the author in [1]. Here we shall extend it to nonconnected groups. A closed subgroup $S$ of $\operatorname{GL}(n, \mathbf{R})$ is said to be semi-algebraic if the factor space $Q(S) / S$ is homeomorphic with a euclidean space $\mathbf{R}^{k}$. In $\S 3$, we shall see that the set $\mathcal{S}$ of all semi-algebraic groups forms an LC-family.

Let $G$ be a connected Lie group, and $\mathcal{G}$ its Lie algebra. Then for $g \in G$, the inner automorphism $G \ni x \mapsto g x g^{-1} \in G$ gives rise to an automorphism $\operatorname{Ad}(g)$ of $\mathcal{G}$, and we have a representation (the adjoint representation)

$$
G \ni g \mapsto \operatorname{Ad}(g) \in \operatorname{GL}(\mathcal{G}) \text {. }
$$

If the adjoint group $\operatorname{Ad}(G)$ is semi-algebraic, $G$ is said to be adjoint semialgebraic. In [1], the author proved that for any connected Lie group $G$, there exists an adjoint semi-algebraic group $S$ containing $G$ as a closed normal subgroup. Then by the nature of our problems, it suffices to consider only adjoint semi-algebraic groups instead of considering general connected Lie groups.

Let $G$ be an adjoint semi-algebraic group. We shall denote $\rho(g)=\operatorname{Ad}(g)$ 
for $g \in G$. A subgroup $H$ of $G$ is called an sa-group if

(1) $\rho(H)$ is semi-algebraic, and

(2) $H$ is an open subgroup of $\rho^{-1} \rho(H)$.

In $\S 4$, we shall see that the set of sa-groups in $G$ forms an LC-family.

Special cases of our results have been studied in Pukanszky [6] and Goto [1]. In particular, the last part of Theorem II was given in [1], in a slightly weaker form.

2. Locally compact groups and LC-families of subgroups. First let us recall some results on locally compact Hausdorff spaces and locally compact groups (= locally compact topological groups).

Let $M$ be a Hausdorff space, and let $Q$ be a subset of $M$. If $Q$ is locally compact, then $Q$ is the intersection of a closed set and an open set. If, in particular, $M$ is locally compact, then the converse is also true. Notice that the intersection, but not the union, of finitely many locally compact sets is again locally compact.

Let $G$ be a locally compact group. Then a subgroup $H$ of $G$ is closed if and only if $H$ is locally compact. Suppose that $H$ is a closed subgroup of $G$. Let $M=G / H$ denote the factor space, and $\pi: G \ni g \mapsto g H \in M$ the natural map. Then a subset $Q$ of $M$ is open, closed, or locally compact if and only if $\pi^{-1} Q$ is so.

(2.1) Let $G$ be a topological group, and let $A$ and $B$ be subgroups of $G$. Let $P$ be a locally compact subset of $G$. If $P$ is $(A, B)$-invariant, then $P$ is $(\bar{A}, \bar{B})$-invariant.

Proof. $A P=P$ implies $A \bar{P}=\bar{P}$, and we have that $A(\bar{P}-P)=\bar{P}-P$. But $\bar{P}-P$ is a closed set, since $P$ is locally compact. Hence $\bar{A}(\bar{P}-P)=\bar{P}$ $-P$, and with $\bar{A} \bar{P}=\bar{P}$, we have that $\bar{A} P=P$. In a similar way, $P \bar{B}=P$.

(2.2) Let $G$ be a locally compact group, and let $D$ and $E$ be subgroups of $G$. Suppose that $E$ is a compactly connected, closed subgroup and the identity component $E_{e}$ of $E$ is a normal subgroup of $G$. Then the double coset decomposition

$$
G=\cup E g \overline{D E_{e}}
$$

is the LC-partition of $G$ with respect to $(E, D)$. Furthermore, each $E_{g} \overline{D E}_{e}$ is a closed subset of $G$.

Proof. Let $\sigma: G \ni g \mapsto g E_{e} \in G / E_{e}$ be the natural homomorphism. We put $E g \overline{D E}_{e}=P=P(g)$. Then $\sigma P=\sigma E \cdot \sigma g \cdot \overline{\sigma D}$ is closed, because $\sigma E$ is a compact group. Hence $P=\sigma^{-1} \sigma P$ is closed.

On the other hand, if $Q$ is a locally compact, $(E, D)$-invariant subset containing $g$, then $Q$ is $\left(E, D E_{e}\right)$-invariant, and $Q \supset P(g)$ by $(2.1)$. Hence $P(g)$ is the local closure of $E g D$. 
(2.3) Let $G$ be a locally compact group, and $H$ a subgroup of $G$. If $H$ is compactly or finitely connected, then so is $\bar{H}$.

Proof. Suppose that $H$ is compactly connected. For each $h$ in $H$, we pick a compact neighborhood $U(h)$ of $h$ in the closure $\bar{H}$ of $H$. Then $V(h)=U(h)$ $\cap H$ is a neighborhood of $h$ in $H$. Let $\varphi$ denote the natural homomorphism

$$
H \ni h \mapsto h H_{e} \in H / H_{e} \text {. }
$$

Then $\varphi(V(h))$ is a neighborhood of $h H_{e}$ in $H / H_{e}$. Since $H / H_{e}$ is compact, there exists a finite set $\left\{h_{1}, \ldots, h_{k}\right\} \subset H$ such that $\cup_{i=1}^{k} \varphi\left(V\left(h_{i}\right)\right)=H / H_{e}$, i.e. $\cup_{i=1}^{k} V\left(h_{i}\right) H_{e}=H$. We put $C=\cup_{i=1}^{k} U\left(h_{i}\right)$. Then $C$ is a compact set, and $C \cdot \bar{H}_{e}$ is closed. Since $\bar{H} \supset C \overline{H_{e}} \supset H$, we have $\bar{H}=C \bar{H}_{e}$. But since $\bar{H}_{e}$ is connected, we have $(\bar{H})_{e} \supset \bar{H}_{e}$ and $\bar{H}=C(\bar{H})_{e}$. Hence $\bar{H}$ is compactly connected.

Next, suppose that $H$ is finitely connected, and $H=\cup_{i=1}^{j} a_{i} H_{e}$ is the coset decomposition. Then $\bar{H} \supset \cup_{i=1}^{j} a_{i} \bar{H}_{e} \supset H$, and $\cup_{i=1}^{j} a_{i} \bar{H}_{e}$ is closed. Hence $\bar{H}=\cup_{i=1}^{j} a_{i} \bar{H}_{e}$.

Next, let $G$ be a locally compact, $\sigma$-compact group, and suppose that there exists an LC-family $\mathscr{F}$ of subgroups of $G$, satisfying (1), ., (7) in $\S 1$. Let $A$ and $B$ be subgroups of $G$. First we shall explain our method to study the $A \times B$ action on $G$.

We put $\mathscr{F}(A)=A^{*}$ and $\mathscr{F}(B)=B^{*}$. By (3) and (4), for any $g$ in $G$, we have that $A^{*}\left(g B^{*} g^{-1}\right)$ is locally compact, and so is $A^{*} g B^{*}$. Hence the double coset decomposition

$$
G=\bigcup_{g \in G} A^{*} g B^{*}
$$

is a partition of $G$ into locally compact, $(A, B)$-invariant subsets. In order to study the $A \times B$ action on each $A^{*} g B^{*}$, we need the following known theorem, see e.g. Helgason [4].

(2.4) Let $L$ be a locally compact, $\sigma$-compact group, and let $M$ be a locally compact Hausdorff space. Suppose that $L$ acts on $M$ transitively. For $m$ in $M$, let $L_{m}$ denote the isotropy subgroup at $m: L_{m}=\{x \in L ; x m=m\}$. Then the map $\xi: L \ni x \mapsto x m \in M$ is (continuous and) open and gives rise to a homeomorphism between the factor space $L / L_{m}$ and $M$.

Since $A^{*}$ and $B^{*}$ are closed subgroups of $G$, they are $\sigma$-compact, and so is $A^{*} \times B^{*}$. On the other hand, $A^{*} \times B^{*}$ acts transitively on $A^{*} g B^{*}$ and the isotropy group $D(g)$ at $g$ is given by

$$
D(g)=\left\{\left(\gamma, g^{-1} \gamma g\right) ; \gamma \in A^{*} \cap g B^{*} g^{-1}\right\} .
$$

By (2.4), the map

$$
\xi=\xi_{g}: A^{*} \times B^{*} \ni(\alpha, \beta) \mapsto \alpha g \beta^{-1} \in A^{*} g B^{*}
$$


induces a homeomorphism between the factor space $\left(A^{*} \times B^{*}\right) / D(g)$ and $A^{*} g B^{*}$.

Let $P$ be a subset of $A^{*} g B^{*}$. If $P$ is locally compact, then so is $\xi^{-1} P$, and conversely. Suppose that $P$ is $(A, B)$-invariant. Then for $(\alpha, \beta) \in \xi^{-1} P$ and $(a, b) \in A \times B$, we have

$$
\xi((a, b)(\alpha, \beta))=a \alpha g \beta^{-1} b \in A P B=P .
$$

Hence $\xi^{-1} P$ is $(A \times B, D(g))$-invariant. Also it is easy to see that if $\xi^{-1} P$ is $(A \times B, D(g))$-invariant, then $P$ is $(A, B)$-invariant. Therefore, $\xi^{-1}$ gives a one-one correspondence between the totality of locally compact, $(A, B)$ invariant subsets of $A^{*} g B^{*}$, and the set of all locally compact, $(A \times B$, $D(g)$ )-invariant subsets of $A^{*} \times B^{*}$.

Suppose that $B$ is closed and consider the map

$$
\eta=\eta_{g}: A^{*} \times B^{*} \ni(\alpha, \beta) \mapsto \alpha g \beta^{-1} B \in A^{*} g B^{*} / B \subset G / B=M .
$$

The map $\eta$ is also continuous and open, and gives rise to a homeomorphism between the totality of double cosets $(\{e\} \times B) \backslash A^{*} \times B^{*} / D(g)$ and $A^{*} g B^{*} / B$.

Before stating the theorem, we shall prove one lemma. For a subgroup $H$ of $G$, we shall denote by $\mathscr{F}_{e}(H)$ the identity component of $\mathscr{F}(H)$.

(2.5) Let $H$ be a compactly connected, closed subgroup of $G$. Then

$$
\mathscr{F}(H)=H \cdot \mathscr{F}\left(H_{e}\right), \quad \mathscr{F}\left(H_{e}\right)=\mathscr{F}_{e}(H) .
$$

$\mathscr{F}(H)$ is compactly connected, and $H_{e}$ is a normal subgroup of $\mathscr{F}(H)$.

Proof. Let $N$ denote the normalizer of $H_{e}$ in $G: N=\left\{g \in G ; g H_{e} g^{-1}=\right.$ $\left.H_{e}\right\}$. Then $\mathscr{F}\left(H_{e}\right) \subset N$ by (6). For $g \in N$, by (3) we have

$$
\mathscr{F}\left(H_{e}\right)=\mathscr{F}\left(g H_{e} g^{-1}\right)=g \mathscr{F}\left(H_{e}\right) g^{-1},
$$

and accordingly $\mathscr{F}\left(H_{e}\right)$ is a normal subgroup of $N$. Since $H \subset N$, we have that $H \cdot \mathscr{F}\left(H_{e}\right)$ is a subgroup of $N$.

Since $H / H_{e}$ is compact, there exists a compact subset $C$ of $H$ such that $H=C H_{e}$. Hence $H \cdot \mathscr{F}\left(H_{e}\right)=C \cdot \mathscr{F}\left(H_{e}\right)$ is a closed subgroup, and we have

$$
H \cdot \mathscr{F}\left(H_{e}\right) / \mathscr{F}\left(H_{e}\right) \cong H / H \cap \mathscr{F}\left(H_{e}\right) .
$$

Since $H \cap \mathscr{F}\left(H_{e}\right) \supset H_{e}$, the factor group $H / H \cap \mathscr{F}\left(H_{e}\right)$ is compact and totally disconnected. Since $\mathscr{F}\left(H_{e}\right)$ is connected by (6), we see that $H \cdot \mathscr{F}\left(H_{e}\right)$ is compactly connected, and the identity component of $H \cdot \mathscr{F}\left(H_{e}\right)$ is $\mathscr{F}\left(H_{e}\right)$. By $(5), H \cdot \mathscr{F}\left(H_{e}\right) \in \mathscr{F}$, and we have $\mathscr{F}(H)=H \cdot \mathscr{F}\left(H_{e}\right)$.

THEOREM III. Let $G$ be a locally compact, o-compact group, with an LCfamily $\mathscr{F}$. Let $A$ and $B$ be subgroups of $G$.

(a) If $A$ and $B$ are compactly connected, then $G$ has an LC-partition with respect to $(A, B)$. 
(b) Suppose that $A$ is a compactly connected, abelian group and B is a closed, connected group. Then for each $g \in G$, we can find a locally compact abelian group $L(g)$, a closed subgroup $L^{\prime}(g)$ of $L(g)$, and a homeomorphism $\varphi$ from $\mathscr{F}(A) g \mathscr{F}(B) / B$ onto $L(g)$, such that the image of the local closure of each orbit $A m, m \in \mathscr{F}(A) g \mathscr{F}(B) / B$, is a coset of $L^{\prime}(g)$.

(c) In (b), if in particular $A$ is a one-parameter subgroup, then the local closure of an orbit Am is homeomorphic either with $\mathbf{R}$ or a certain compact connected abelian group.

Proof of (a). By (2.3), $\bar{A}$ and $\bar{B}$ are compactly connected. Hence after this we can suppose that $A$ and $B$ are closed, by (2.1). Then by (2.5), $A_{e}$ and $B_{e}$ are normal subgroups of $A^{*}=\mathscr{F}(A)$ and $B^{*}=\mathscr{F}(B)$, respectively. Hence $(A \times B)_{e}=A_{e} \times B_{e}$ is a normal subgroup of $A^{*} \times B^{*}$. Since $A \times B$ is compactly connected, we can apply (2.2), and for each $g$ in $G$,

$$
A^{*} \times B^{*}=\cup(A \times B) x \overline{D(g)\left(A_{e} \times B_{e}\right)}
$$

is the LC-partition of $A^{*} \times B^{*}$ with respect to $(A \times B, D(g))$. Hence

$$
A^{*} g B^{*}=\cup \xi_{g}\left((A \times B) x \overline{D(g)\left(A_{e} \times B_{e}\right)}\right)
$$

is a partition of $A^{*} g B^{*}$ into minimal, locally compact, $(A, B)$-invariant subsets. This completes the proof of (a).

Proof of (b) AND (c). By (7) and (6), $A^{*}$ and $B^{*} / B$ are abelian groups. Hence $(A \times B) D(g)$ is a subgroup of $A^{*} \times B^{*}$, and the coset decomposition

$$
A^{*} \times B^{*}=\cup x \overline{(A \times B) D(g)}
$$

gives the LC-partition of $A^{*} \times B^{*}$ with respect to the pair $(A \times B, D(g))$. Hence $G$ has an LC-partition with respect to the pair $(A, B)$.

Since $(\{e\} \times B) D(g)$ is a closed normal subgroup of $A^{*} \times B^{*}$, the set $(\{e\} \times B) \backslash A^{*} \times B^{*} / D(g)$ can be identified with the abelian group $L(g)=$ $A^{*} \times B^{*} /(\{e\} \times B) D(g)$. Thus the map

$$
\eta_{g}: A^{*} \times B^{*} \ni(\alpha, \beta) \mapsto \alpha g \beta^{-1} B \in A^{*} g B^{*} / B
$$

induces a homeomorphism $\eta_{g}^{\prime}$ from $L(g)$ onto $A^{*} g B^{*} / B$.

Let us put $\overline{(A \times B) D(g)} /(\{e\} \times B) D(g)=L^{\prime}(g)$. Then for each $x \in A^{*}$ $\times B^{*}$, we put $x^{\#}=x(\{e\} \times B) D(g) \in L(g)$, and get that

$$
\eta_{g}^{\prime}\left(x^{\sharp} L^{\prime}(g)\right)=\eta_{g} \overline{(x(A \times B) D(g))}
$$

is the local closure of the orbit $A \eta_{g}(x)$.

If, in particular, $A$ is a one-parameter subgroup, then $(A \times B) D(g) /(\{e\}$ $\times B) D(g)$ is a one-parameter subgroup of $L(g)$, and is dense in $L^{\prime}(g)$. Hence $L^{\prime}(g)$ is either $\mathbf{R}$ or compact. This completes the proof of (b) and (c).

ReMARK. Let $L$ be a locally compact, $\sigma$-compact group, and $G$ a closed 
subgroup of $L$. Let $A$ and $B$ be subgroups of $G$. If $L$ has an LC-family $\mathscr{F}$, then Theorem III, except for the part concerning $\mathscr{F}$-hulls, holds for $G$. In fact, if $P_{\lambda}$ is the local closure of $A g B$ in $L$ for $g \in G$, then $P_{\lambda} \cap G$ is locally compact, $(A, B)$-invariant, and coincides with $P_{\lambda}$. Hence $P_{\lambda} \subset G$.

3. Semi-algebraic groups. In this section we shall study subgroups of $\mathrm{GL}(n, \mathbf{R})$. A subgroup $H$ of $\mathrm{GL}(n, \mathbf{R})$ is said to be pre-algebraic if $H$ is an open subgroup of a suitable algebraic group. Since an algebraic group is finitely connected, so is any pre-algebraic group. Let $\mathbb{Q}$ denote the set of all pre-algebraic groups in $\operatorname{GL}(n, \mathbf{R})$. The following theorem is known; in particular, the proof of (4) for $\mathbb{Q}$ can be found in [2].

(3.1) THEOREM. $\mathcal{Q}$ is an LC-family in $\mathrm{GL}(n, \mathbf{R})$. Any member of $\mathbb{Q}$ is finitely connected.

For a subgroup $H$ of $\mathrm{GL}(n, \mathbf{R})$, let $\mathbb{Q}_{e}(H)$ denote the identity component of $\mathbb{Q}(H)$.

(3.2) (1) For any subgroup $H$ of $\mathrm{GL}(n, \mathbf{R}), H \cdot \mathbb{Q}_{e}(H)=\mathbb{Q}(H)$.

(2) If in particular $H$ is finitely connected, then $\mathbb{Q}_{e}(H)=\mathbb{Q}\left(H_{e}\right)$.

Proof. (1) Since $\mathbb{Q}_{e}(H)$ is a normal subgroup of $\mathcal{Q}(H)$, we have that $H \cdot \mathbb{Q}_{e}(H)$ is a subgroup of $\mathbb{Q}(H)$. Since $\mathbb{Q}_{e}(H)$ is contained in $H \cdot \mathbb{Q}_{e}(H)$, we see that $H \cdot \mathbb{Q}_{e}(H)$ is open in $\mathbb{Q}(H)$, and is pre-algebraic. Hence $H$. $\mathbb{Q}_{e}(H)=\mathbb{Q}(H)$.

(2) It is obvious that $\bar{H}_{e}$ is the identity component of $\bar{H}$. Hence it reduces to (2.5).

Let $H$ be a closed connected subgroup of $\operatorname{GL}(n, \mathbf{R})$. Then $H$ is normal in $\mathcal{Q}(H)$, and the factor group $\mathcal{Q}(H) / H$ is a connected abelian group by (6). Since $\mathcal{Q}(H) / H$ is a Lie group, there exist nonnegative integers $k$ and $h$ such that $\mathbb{Q}(H) / H=\mathbf{R}^{k} \times(\mathbf{R} / \mathbf{Z})^{h}$. Let $K$ be a maximal compact subgroup of $\mathbb{Q}(H)$. Then $K H / H$ is a maximal compact subgroup of $\mathbb{Q}(H) / H$; see Iwasawa [5].

In [2], the author defined $H$ to be semi-algebraic if $H$ contains all compact subgroups of $\mathcal{Q}(H)$. This is equivalent to saying that the factor group $\mathbb{Q}(H) / H$ is isomorphic with the vector group $\mathbf{R}^{k}$, or that $\mathcal{Q}(H) / H$ is homeomorphic with the euclidean space $\mathbf{R}^{k}$. Now let us extend the definition to nonconnected groups.

Definition 5. A closed subgroup $H$ of $\operatorname{GL}(n, \mathbf{R})$ is said to be semi-algebraic if the factor space $\mathcal{Q}(H) / H$ is homeomorphic with a euclidean space.

REMARK AND CORRECTION. In [2], the author defined nonconnected semialgebraic groups in a more restrictive manner. However, he later found the new definition more convenient. In the Proposition, p. 72 in [2], "and conversely." must be removed.

(3.3) Let $G$ be a Lie group, and $H$ a closed subgroup of $G$. If the factor 
space $G / H$ is connected and simply connected, then

$$
H \cap G_{e}=H_{e} \text { and } G / G_{e} \cong H / H_{e} \text {. }
$$

Proof. Since $G / H$ is connected, we have that $G_{e} H=G$ and $G / H \sim$ $G_{e} / H \cap G_{e}$, where $\sim$ denotes the existence of a homeomorphism. Since $G / H$ is simply connected, $H \cap G_{e}$ is connected and coincides with $H_{e}$. Hence $G / G_{e}=H G_{e} / G_{e} \cong H / H \cap G_{e}=H / H_{e}$.

(3.4) If $S$ is semi-algebraic, then $S$ is finitely connected and $S_{e}$ is semialgebraic, and vice versa.

Proof. If $S$ is semi-algebraic, then by (3.3), $S / S_{e} \cong \mathbb{Q}(S) / \mathbb{Q}_{e}(S)$ is finite and $\mathbb{Q}_{e}(S) \cap S=S_{e}$. Hence by (3.2),

$$
\mathbb{Q}(S) / S=S \cdot \mathbb{Q}_{e}(S) / S \sim \mathbb{Q}_{e}(S) / \mathbb{Q}_{e}(S) \cap S=\mathbb{Q}\left(S_{e}\right) / S_{e},
$$

and $\mathcal{Q}\left(S_{e}\right) / S_{e}$ is homeomorphic with a euclidean space.

Conversely, suppose that $S$ is finitely connected and $S_{e}$ is semi-algebraic. Then $\left(\mathbb{Q}_{e}(S) \cap S\right) / S_{e}$ is a subgroup of $\mathbb{Q}_{e}(S) / S_{e}=\mathbb{Q}\left(S_{e}\right) / S_{e} \cong \mathbf{R}^{k}$. Since $\left(\mathscr{Q}_{e}(S) \cap S\right) / S_{e}$ is a finite group, it must reduce to the identity and $\mathbb{Q}_{e}(S) \cap$ $S=S_{e}$. Then

$$
\begin{aligned}
\mathbb{Q}(S) / S & =S \cdot \mathbb{Q}_{e}(S) / S_{e}=S \cdot \mathbb{Q}\left(S_{e}\right) / S_{e} \sim \mathbb{Q}\left(S_{e}\right) /\left(\mathbb{Q}\left(S_{e}\right) \cap S\right) \\
& =\mathbb{Q}\left(S_{e}\right) / S_{e} \cong \mathbf{R}^{k} .
\end{aligned}
$$

Let $G$ be a countably connected Lie group. A subgroup $H$ of $G$ is said to be a Lie subgroup if there exists a countably connected Lie group $H^{*}$ and a continuous one-one homomorphism $f$ from $H^{*}$ into $G$ such that $f\left(H^{*}\right)=H$. A closed subgroup is a Lie subgroup. Let $H$ be a Lie subgroup of $G$. Then the Lie group $H^{*}$ is uniquely determined up to topological isomorphisms. $H$ is called a connected, or a finitely connected, Lie subgroup if $H^{*}$ is connected, or finitely connected, respectively. $H$ is a connected Lie subgroup if and only if $H$ is arcwise connected. If $H_{1}$ and $H_{2}$ are Lie subgroups and if $H_{1} H_{2}$ is a subgroup, then $\mathrm{H}_{1} \mathrm{H}_{2}$ is a Lie subgroup.

Let us denote the set of all semi-algebraic groups in GL $(n, R)$ by $\delta$.

(3.5) Let $\left\{S_{\lambda}\right\}$ be a subset of $\delta$. Then the intersection $\cap S_{\lambda}$ is semialgebraic.

Proof. Since any semi-algebraic group is finitely connected, $\delta$ satisfies the descending chain condition. Hence it suffices to prove that $A \cap B \in \mathcal{S}$ for $A$ and $B$ in $\mathcal{S}$. We put

$$
A_{1}=\mathscr{Q}\left(A_{e}\right), \quad B_{1}=\mathscr{Q}\left(B_{e}\right) \text { and } C_{1}=A_{1} \cap B_{1} .
$$

Then $C_{1}$ is pre-algebraic and is finitely connected. Therefore $A_{e} C_{1}$ is a finitely connected Lie subgroup of $A_{1}$, and so is $A_{e} C_{1} / A_{e}$ in $A_{1} / A_{e}$. Since $A_{1} / A_{e}$ is a vector group, so is $A_{e} C_{1} / A_{e}$. Thus $C_{1} / C_{1} \cap A_{e} \cong A_{e} C_{1} / A_{e}$ is a vector group. By (3.3), $C_{1} \cap A_{e}$ is finitely connected since $C_{1}$ is. In a similar way, $C_{1} / C_{1} \cap$ 
$B_{e}$ is a vector group and $C_{1} \cap B_{e}$ is finitely connected. It, then, follows that $\left(C_{1} \cap A_{e}\right)\left(C_{1} \cap B_{e}\right)$ is a finitely connected Lie subgroup in $C_{1}$, and so is $\left(C_{1} \cap A_{e}\right)\left(C_{1} \cap B_{e}\right) / C_{1} \cap B_{e}$ in $C_{1} / C_{1} \cap B_{e}=\mathbf{R}^{k}$. Hence

$$
\left(C_{1} \cap A_{e}\right)\left(C_{1} \cap B_{e}\right) / C_{1} \cap B_{e} \cong C_{1} \cap A_{e} / C
$$

is a vector group, where $C=A_{e} \cap B_{e}=\left(C_{1} \cap A_{e}\right) \cap\left(C_{1} \cap B_{e}\right) . C_{1} \cap A_{e}$ being finitely connected, so is $C$ by (3.3). Moreover, $C$ is of finite index in $A \cap B$ since $A$ and $B$ are finitely connected. Therefore $A \cap B$ is finitely connected.

Next, we shall prove that $(A \cap B)_{e}$ is semi-algebraic. Let $K$ be a compact connected subgroup of $C_{1}$. Then $K \subset A_{1}$, and $K \subset A$. Similarly $K \subset B$. Hence $K \subset(A \cap B)_{e}$. That is, any maximal compact subgroup of $\left(C_{1}\right)_{e}$ is contained in $(A \cap B)_{e}$. It follows from $\left(C_{1}\right)_{e}$ being pre-algebraic that $(A \cap$ $B)_{e}$ is semi-algebraic.

(3.6) Let $A$ and $B$ be in $\delta$. Then the double coset $A B$ is locally compact. ${ }^{1}$

Proof. As before, we put $\mathbb{Q}(A)=A^{*}, \mathbb{Q}(B)=B^{*}$ and

$$
D=\left\{(\gamma, \gamma) ; \gamma \in A^{*} \cap B^{*}\right\} \subset A^{*} \times B^{*} .
$$

Since $A^{*} B^{*}$ is locally compact, the map

$$
\xi: A^{*} \times B^{*} \ni(\alpha, \beta) \rightarrow \alpha \beta^{-1} \in A^{*} B^{*}
$$

is continuous and open, and gives rise to a homeomorphism between the factor space $A^{*} \times B^{*} / D$ and $A^{*} B^{*}$. Hence it suffices to prove that $\xi^{-1}(A B)$ $=(A \times B) D$ is closed in $A^{*} B^{*}$.

Since $A, B$ and $D$ are all finitely connected, $(A \times B) D$ is a finite union of the sets of the form

$$
\varepsilon\left(A_{e} \times B_{e}\right) D_{e} \delta, \quad \varepsilon \in A \times B, \delta \in D .
$$

On the other hand, $\left(A_{e} \times B_{e}\right) D_{e}$ is a connected Lie subgroup of $\left(A^{*} \times B^{*}\right)_{e}$ containing all compact subgroups, and is closed.

From (3.1), (3.4), (3.5) and (3.6), we have the following theorem:

(3.7) THEOREM. $\mathcal{S}$ is an LC-family in $\operatorname{GL}(n, \mathbf{R})$, and any member of $\mathcal{S}$ is finitely connected.

(3.8) Let $\varphi$ be a rational homomorphism from an algebraic group $A$ into $\mathrm{GL}(j, \mathbf{R})$. Let $S$ be a semi-algebraic group in $A$. Then $\varphi(S)$ is semi-algebraic.

Proof. Since $S$ is finitely connected, so is $\varphi(S)$. Hence it suffices to prove that $\varphi\left(S_{e}\right)$ is semi-algebraic. Therefore, we may suppose that $S$ is connected.

Let $N$ be the kernel of $\varphi$ restricted to $\mathcal{Q}(S)$. Then $N$ is pre-algebraic, and is finitely connected. Hence $N S / S$ is a finitely connected Lie subgroup of $\mathscr{Q}(S) / S$. Since $\mathscr{Q}(S) / S$ is a vector group, so is $N S / S$, and $N S$ is closed and

${ }^{1}(3.6)$ was proved in [2] under a slightly stronger condition. 
connected. Hence $\varphi(S)=\varphi(N S)$ is closed in $\varphi \mathbb{Q}(S)$. Since $\varphi \mathbb{Q}(S)=\mathbb{Q} \varphi(S)$, see [3], we have

$$
\mathbb{Q} \varphi(S) / \varphi(S)=\varphi \mathbb{Q}(S) / \varphi(S) \cong \mathbb{Q}(S) / N S .
$$

Thus, recalling $\mathscr{Q}(S) / N S=\mathbb{Q}(S) / S / N S / S$ is a vector group, it follows that $\varphi(S)$ is semi-algebraic.

4. sa-groups in an adjoint semi-algebraic group. Let $G$ be a connected Lie group, $\mathcal{G}$ its Lie algebra, and let $\rho$ denote the adjoint representation of $G$ :

$$
G \ni g \mapsto \rho(g)=\operatorname{Ad}(g) \in \operatorname{Ad}(\mathcal{G}) \subset \operatorname{GL}(\mathcal{G}) .
$$

The kernel of $\rho$ is the center $Z$ of $G$. The connected Lie group $G$ is said to be adjoint semi-algebraic if the adjoint $\operatorname{group} \operatorname{Ad}(G)=\operatorname{Ad}(\mathcal{G})$ is semi-algebraic. By (3.8), a connected semi-algebraic group is adjoint semi-algebraic, and the converse is given by the following:

(4.1) Let $G$ be an adjoint semi-algebraic group. Then there exists a semi-algebraic group $G^{\prime} \subset \mathrm{GL}(n, \mathbf{R})$, for a sufficiently large $n$, such that $G$ is locally isomorphic with $G^{\prime}$.

(4.1) was proved in [2], along with (4.2).

(4.2) Let $G$ be a connected Lie group. Then there exists an adjoint semi-algebraic group $S$ containing $G$ as a closed normal subgroup.

After this, we assume that $G$ is an adjoint semi-algebraic group, $\mathcal{G}$ its Lie algebra, $\rho$ the adjoint representation of $G$ and $Z$ is the center of $G$.

Definition 6. A subgroup $H$ of $G$ is said to be an sa-group if

(i) $\rho(H)$ is semi-algebraic, and

(ii) $H$ is open in $\rho^{-1} \rho(H)$.

If $H$ is an sa-group, then

(ii') $H$ is closed and $H \supset Z_{e}$.

Conversely, (i) and (ii') imply (ii) obviously. Let $\mathfrak{g}$ denote the set of all sa-groups in $G$.

(4.3) Let $S$ be an sa-group.

(1) If $S^{\prime}$ is an open subgroup of $S$, then $S^{\prime}$ is sa.

(2) If $S^{\prime \prime}$ contains $S$ as a subgroup of finite index, then $S^{\prime \prime}$ is an sa-group.

PROOF. If $S$ is an sa-group, then $\rho(S)$ is semi-algebraic and

$$
\rho\left(S^{\prime \prime}\right) \supset \rho(S) \supset \rho\left(S^{\prime}\right) \supset \rho\left(S_{e}\right)=\rho\left(S^{\prime \prime}\right)_{e} \text {. }
$$

Hence $\rho\left(S^{\prime}\right)$ is open in $\rho(S)$, and is semi-algebraic. Also $\rho(S)$ is of finite index in $\rho\left(S^{\prime \prime}\right)$, and $\rho\left(S^{\prime \prime}\right)$ is semi-algebraic.

Next, $S^{\prime}$ and $S^{\prime \prime}$ are closed subgroups and contain $S_{e} \supset Z_{e}$. Hence $S^{\prime}$ and $S^{\prime \prime}$ are sa-groups.

(4.4) If $\left\{S_{\lambda} ; \lambda \in \Lambda\right\}$ is a nonempty subset of $\mathfrak{g}$, then $\cap S_{\lambda} \in \mathfrak{S}$. 
Proof. We put $S_{\lambda}^{\prime}=S_{\lambda} Z=\rho^{-1} \rho\left(S_{\lambda}\right)$ for $\lambda \in \Lambda$. Then it is obvious that

$$
\cap \rho\left(S_{\lambda}^{\prime}\right)=\rho\left(\cap S_{\lambda}^{\prime}\right) \text { and } \rho^{-1} \rho\left(\cap S_{\lambda}^{\prime}\right)=\cap S_{\lambda}^{\prime}
$$

Since $\rho\left(S_{\lambda}^{\prime}\right)=\rho\left(S_{\lambda}\right)$ is semi-algebraic, so is $\cap \rho\left(S_{\lambda}^{\prime}\right)$. Hence $\cap S_{\lambda}^{\prime}$ is an sa-group.

Next, since $S_{\lambda}$ is open in $S_{\lambda}^{\prime}$, the identity components of the two groups coincide, and $\left(\cap S_{\lambda}\right)_{e}=\left(\cap S_{\lambda}^{\prime}\right)_{e}$. Therefore $\cap S_{\lambda}^{\prime} \supset \cap S_{\lambda} \supset\left(\cap S_{\lambda}^{\prime}\right)_{e}$, and $\cap S_{\lambda}$ is an open subgroup of $\cap S_{\lambda}^{\prime}$. By (4.3), $\cap S_{\lambda}$ is an sa-group.

By (4.4), for any subgroup $H$ of $G$, there corresponds the $\mathfrak{s}$-hull $\mathfrak{s}(H)$, the smallest sa-group containing $H$. Let $\mathfrak{J}_{e}(H)$ denote the identity component of $\mathfrak{S}(H)$.

(4.5) (1) If $H$ is a connected subgroup of $G$, then

$$
\mathfrak{s}(H)=\left(\rho^{-1} \delta \rho(H)\right)_{e} .
$$

(2) If $H$ is a finitely connected, closed subgroup of $G$, then

$$
\mathfrak{s}(H)=H \cdot \mathfrak{g}_{e}(H), \quad \mathfrak{g}_{e}(H)=\mathfrak{s}\left(H_{e}\right),
$$

$\mathfrak{S}(H)$ is finitely connected, and $H_{e}$ is a normal subgroup of $\mathfrak{s}(H)$.

Proof. (1) We put $S=\left(\rho^{-1} \delta \rho(H)\right)_{e}$. Then

$$
\rho(S)=\delta \rho(H) \text { and } \rho^{-1} \rho(S)_{e}=S,
$$

and $S$ is an sa-group.

Next, suppose that $T$ is an sa-group containing $H$. Then

$$
\rho(T) \supset \delta \rho(H) \text { and } \rho^{-1} \rho(T) \supset \rho^{-1} \delta \rho(H) \supset S .
$$

Since $T$ is open in $\rho^{-1} \rho(T)$ and $S$ is connected, we have that $T \supset S$.

(2) Let $\mathcal{H}$ be the Lie algebra of $H$, and let $N$ be the normalizer of $H_{e}$ in $G$. Then $M=\{x \in \mathrm{GL}(\mathcal{G}) ; x \mathcal{H}=\mathscr{H}\}$ is an algebraic group, and $\rho(N)=$ $\operatorname{Ad}(\mathcal{G}) \cap M$ is a semi-algebraic group in $\operatorname{GL}(\mathcal{G})$. Hence

$$
\rho(N) \supset \delta \rho\left(H_{e}\right) \text { and } N=\rho^{-1} \rho(N) \supset \rho^{-1} \delta \rho\left(H_{e}\right) \text {. }
$$

Since $\rho\left(H_{e}\right)$ is a normal subgroup of $\rho(N)$, for $x \in \rho(N)$

$$
\delta \rho\left(H_{e}\right)=\delta\left(x \rho\left(H_{e}\right) x^{-1}\right)=x \delta \rho\left(H_{e}\right) x^{-1},
$$

and $\delta \rho\left(H_{e}\right)$ is a normal subgroup of $\rho(N)$. Hence $N$ normalizes $\rho^{-1} \delta \rho\left(H_{e}\right)$, and its identity component $\mathfrak{g}\left(H_{e}\right)$. Since $H$ is contained in $N, H \cdot \mathfrak{s}\left(H_{e}\right)$ is a subgroup of $N$. By $\mathfrak{s}\left(H_{e}\right) \supset H_{e}, \mathfrak{s}\left(H_{e}\right)$ is of finite index in $H \cdot \mathfrak{s}\left(H_{e}\right)$. Hence $H \cdot \mathfrak{s}\left(H_{e}\right)$ is an sa-group, by (4.3) (2), and $\mathfrak{s}(H)=H \cdot \mathfrak{s}\left(H_{e}\right)$. Thus $\mathfrak{s}\left(H_{e}\right)$ is a closed connected subgroup of finite index in $\mathfrak{s}(H)$, and so $\mathfrak{Z}_{e}(H)=\mathfrak{S}\left(H_{e}\right)$. That $N \supset \mathfrak{s}(H)$ implies that $H_{e}$ is normal in $\mathfrak{s}(H)$.

For a group $L$, let $[L, L]$ denote the commutator subgroup of $L$. If, in particular, $L$ is a connected Lie group with Lie algebra $\mathcal{L}$, then $[L, L]$ is a connected Lie subgroup of $L$ and the Lie algebra of $[L, L]$ is $[\mathcal{L}, \mathcal{E}]$. 
(4.6) Let $H$ be a connected Lie subgroup of $G$. Then $[\mathfrak{s}(H), \mathfrak{s}(H)]=$ $[H, H]$.

Proof. First suppose that $G$ is a semi-algebraic group in $\operatorname{GL}(n, \mathbf{R})$. We put $S=\delta(H) Z$. Then $[S, S]=[\delta(H), \delta(H)]=[H, H]$. By $(3.8), \rho(S)=$ $\rho \delta(H)$ is semi-algebraic. Hence $S=\rho^{-1} \rho(S)$ is an sa-group, and $S \supset \mathfrak{S}(H)$. Therefore $[S, S] \supset[\mathfrak{s}(H), \mathfrak{s}(H)] \supset[H, H]$ and $[\mathfrak{s}(H), \mathfrak{s}(H)]=[H, H]$.

Now, we shall consider the general case. By (4.1), there exists a connected semi-algebraic group $G^{\prime}$ which is locally isomorphic with $G$. Let us identify $\mathcal{G}$ with the Lie algebra of $G^{\prime}$. Let $\mathcal{H}$ be the Lie algebra of $H$, and let $H^{\prime}$ be the connected Lie subgroup of $G^{\prime}$ corresponding to the Lie algebra $\mathcal{H}$. Then by (4.5) (1), the Lie algebra of $\mathfrak{S}\left(H^{\prime}\right)$ coincides with the Lie algebra $\mathfrak{S}(\mathcal{H C})$ of $\mathfrak{I}(H)$. Thus $\left[\mathfrak{S}\left(H^{\prime}\right), \mathfrak{S}\left(H^{\prime}\right)\right]=\left[H^{\prime}, H^{\prime}\right]$ implies $[\mathfrak{S}(\mathcal{H C}), \mathfrak{s}(\mathcal{F})]=[\mathfrak{H}, \mathfrak{H}]$, whence $[\mathfrak{s}(H), \mathfrak{s}(H)]=[H, H]$.

(4.7) If $H$ is a finitely connected, abelian subgroup of $G$, then $\Xi(H)$ is abelian.

Proof. Because $\overline{H Z_{e}}$ is finitely connected and abelian, we can suppose that $H$ is closed and $H \supset Z_{e}$, without loss of generality. By (4.5) (2),

$$
\mathfrak{s}(H)=H \cdot \mathfrak{g}_{e}(H), \quad \mathfrak{g}_{e}(H)=\mathfrak{s}\left(H_{e}\right),
$$

where $\mathfrak{S}_{e}(H)$ is abelian, by (4.6).

Let $A$ be an abelian group in $\operatorname{GL}(n, \mathbf{R})$, and let $C$ be the center of the centralizer of $A$. Then $C$ is an abelian algebraic group and $C \supset A$. Therefore $\mathcal{Q}(A)$ is abelian, and so is $\mathcal{S}(A)$. That is, the $\mathcal{S}$-hull of any abelian group is abelian. Since $H$ is abelian, so is $\rho(H)$, and $\delta \rho(H)$ is an abelian group. Hence $[\mathfrak{s}(H), \mathfrak{S}(H)] \subset Z$.

Let $\mathcal{H}$ and $\mathfrak{S}(\mathcal{H})$ denote the Lie algebra of $H$ and $\mathfrak{S}(H)$, respectively. $\mathfrak{S}_{e}(H)$ being a normal subgroup of $\mathfrak{s}(H), \rho(H)$ leaves $\mathfrak{B}(\mathcal{H})$ invariant. For $h \in H$, let $\mu(h)$ denote the restriction of $\rho(h)$ to $\mathfrak{g}(\mathcal{H C})$. Since $\mathfrak{S}_{e}(H)$ is abelian and $H_{e} \subset \mathfrak{S}_{e}(H)$, the kernel of $\mu$ contains $H_{e}$, and $\mu$ induces a representation of the finite group $H / H_{e}$. Therefore, the representation $\mu: H \rightarrow \operatorname{GL}(\mathfrak{B}(\mathcal{H C}))$ is completely reducible. Owing to $\mu(H) \mathcal{H}=\mathcal{H}$, we can find a subspace (subalgebra) $\mathfrak{K}$ of $\mathfrak{s}(\mathcal{H})$ such that $\mathfrak{s}(\mathcal{H})=\mathscr{H} \oplus \mathfrak{K}, \mu(H) \mathfrak{K}=\mathfrak{N}$. For any $h$ in $H$, we have $(\mu(h)-1) \mathcal{H}=0$ because $H$ is abelian, and $(\mu(h)-$ $1) \mathfrak{s}(\mathcal{H}) \subset \mathfrak{N}$.

On the other hand, for any $X \in \mathfrak{s}(\mathcal{H})$ and the real parameter $t$,

$$
a(t)=h(\exp t X) h^{-1}(\exp (-t X))=\exp \left(t(\mu(h)-1) X+O\left(t^{2}\right)\right)
$$

is a curve in $Z$, and belongs to $Z_{e}$. Since $Z_{e} \subset H$ and the tangent vector to $a(t)$ at $t=0$ is $(\mu(h)-1) X$, we have that $(\mu(h)-1) X \in \mathcal{K}$, and $(\mu(h)-1) \mathfrak{s}(\mathcal{H}) \subset \mathcal{H}$. It follows that $(\mu(h)-1) \mathfrak{s}(\mathcal{H})=0$ since $\mathcal{H} \cap \mathfrak{T}=$ $\{0\}$. Hence $h$ commutes with every element of $\mathfrak{J}_{e}(H)$. Therefore $\mathfrak{s}(H)=H$. $\mathfrak{g}_{e}(H)$ is abelian. 
REMARK. The semi-algebraic hull of an abelian group is abelian as we saw in the proof above. But this is not true for the 3 -hull. For example,

$$
G=\left\{g(x, y, z)=\left(\begin{array}{cccc}
e^{x} & 0 & 0 & 0 \\
0 & \cos x & \sin x & y \\
0 & -\sin x & \cos x & z \\
0 & 0 & 0 & 1
\end{array}\right) ; x, y, z \in \mathbf{R}\right\}
$$

is an adjoint (semi-)algebraic group, and

$$
H=\{g(x, y, z) ; x \in 2 \pi \mathbf{Z}, y \in \mathbf{Z}, z \in \mathbf{Z}\}
$$

is an abelian subgroup of $G$, but $\mathfrak{s}(H)=G$ is not abelian.

Thus we have

(4.8) THEOREM. Let $G$ be an adjoint semi-algebraic group, and let $\$$ be the totality of sa-groups in G. Then $s$ is an LC-family.

ADDED IN PROOF. The author learned from Philip Green that he had proved in his unpublished paper that any connected Lie group can be embedded as a closed normal subgroup in a suitable Lie group whose adjoint group is pre-algebraic. By his theorem, the main results of this paper can be established without semi-algebraic groups. But the author thinks the theory of semi-algebraic groups itself has some significance, so he leaves the paper in the original form.

\section{BIBLIOGRAPHY}

1. M. Goto, Orbits of one-parameter groups. III. Lie group case, J. Math. Soc. Japan 23 (1971), 95-102.

2. __ Products of two semi-algebraic groups, J. Math. Soc. Japan 25 (1973), 71-74.

3. M. Goto and H. C. Wang, Non-discrete uniform subgroups of semisimple Lie groups, Math. Ann. 198 (1972), 259-286.

4. S. Helgason, Differential geometry and symmetric spaces, Academic Press, New York, 1962.

5. K. Iwasawa, On some types of topological groups, Ann. of Math. 50 (1949), 507-558.

6. L. Pukanszky, Unitary representations of solvable Lie groups, Ann. Sci. Ecole Norm. Sup. 4 (1971), 457-608.

Department of Mathematics, University of Pennsylvania, Philadelphia, Pennsyluania 19174 